Article

\title{
Approximate Solutions for Fractional Boundary Value Problems via Green-CAS Wavelet Method
}

\author{
Muhammad Ismail $^{1}\left(\mathbb{D}\right.$, Umer Saeed $^{2}$, Jehad Alzabut ${ }^{3, *(1)}$ and Mujeeb ur Rehman ${ }^{1}$ \\ 1 School of Natural Sciences, National University of Sciences and Technology, Islamabad 44000, Pakistan; \\ m.ismail0075@gmail.com (M.I.); mrehman@sns.nust.edu.pk (M.u.R.) \\ 2 NUST Institute of Civil Engineering, School of Civil and Environmental Engineering, National University of \\ Sciences and Technology, Islamabad 44000, Pakistan; umer.math@gmail.com \\ 3 Department of Mathematics and General Sciences, Prince Sultan University, Riyadh 11586, Saudi Arabia \\ * Correspondence: jalzabut@psu.edu.sa
}

Received: 21 September 2019; Accepted: 11 November 2019; Published: 2 December 2019

check for updates

\begin{abstract}
In this study, we present a novel numerical scheme for the approximate solutions of linear as well as non-linear ordinary differential equations of fractional order with boundary conditions. This method combines Cosine and Sine (CAS) wavelets together with Green function, called Green-CAS method. The method simplifies the existing CAS wavelet method and does not require conventional operational matrices of integration for certain cases. Quasilinearization technique is used to transform non-linear fractional differential equations to linear equations and then Green-CAS method is applied. Furthermore, the proposed method has also been analyzed for convergence, particularly in the context of error analysis. Sufficient conditions for the existence of unique solutions are established for the boundary value problem under consideration. Moreover, to elaborate the effectiveness and accuracy of the proposed method, results of essential numerical applications have also been documented in graphical as well as tabular form.
\end{abstract}

Keywords: Green-CAS method; CAS wavelets; Caputo integration and derivative; fractional differential equations; collocation points

\section{Introduction}

Fractional calculus, which is the generalization of ordinary integration and differentiation, has become an essential component in recipes for genre of science and engineering. This significance of fractional calculus is its essence of being a potential tool with higher accuracy and concluding precise results in different areas. The distinctive feature of fractional operators is that the fractional differential operators are of non-local character. Hence, the subject takes history along with non-local distributed effects into account [1-3]. Several problems of physical nature are central to, and crux of fractional differential equation. Recently many researchers have focused on techniques and methods for solving fractional differential equations [4-10]. In essence, fractional calculus has been deployed for fluid dynamics [11], bio engineering [12], electromagnetism [13], modeling the transfer of heat in heterogeneous media [14] and anomalous diffusion [15,16]. The manipulative control actions of human operators interacting with fractional-order plants are studied in [17]. The authors introduced a generalized fractional crossover model, which is justified and validated with the experimental data. In [18], a model is developed by generalizing the crossover model which characterizing the human control of systems with both integer and fractional-order plant dynamics. Present work has examined the characteristics of human control using fractional-order dynamics.

Wavelet analysis is well established and successful mathematical tool with numerous applications in engineering and science. Wavelets consists of expressions of functions expanded as summation 
of basic functions. These basic functions are achieved by translation and compression of a mother wavelet function. Thus, inheriting properties of locality and smoothness, which makes it easy to study the properties of integer and locality in the process of expressing function [19]. Wavelet has stirred considerable interest in applying them for numerical solutions of classical ordinary and partial differential equations. Recently, researcher have successfully extended some standard wavelet methods for numerical solutions for fractional differential equations as well. It is somehow surprising that among different solution techniques, the Cosine and Sine (CAS) wavelet method have rather less attention. CAS wavelet technique have been used to approximate solutions for Fredholm integral equations [20], and integro-differential equations [20-22]. Moreover, CAS wavelet operational matrices have been used to approximate solutions of non-linear Volterra integro-differential equations [23]. The CAS Picard methods has been used to approximate the solutions of non-linear fractional differential equations [24]. Other applications of some wavelet methods in the area of applied mathematics include numerical integration, numerical solutions of fractional ordinary and fractional partial differential equations [25-28]. Meanwhile, different types of wavelets are used such as the Haar wavelet [29,30], B-spline [31], Daubechies [32] and Legendre wavelet [33,34].

The application of Chebyshev wavelets method for numerical solutions of fractional fourth, sixth and eighth order linear and non-linear boundary value problems is considered in [35]. The reproducing kernel Hilbert space method is used in [36] for the solutions of fourth order fractional boundary value problems. In [37], authors constructed a new pseudospectral integration matrix of any arbitrary order $a \in \mathbb{R}^{+}$for the solution of fractional initial and boundary value problems. They used the Chebyshev interpolating polynomial for functions at the Gauss-Lobatto points in $[-1,1]$. A novel approach is introduced in [38] for the solution of fractional initial and boundary-values problems. Solution is approximated by the truncated series of fractional powers of the independent variables.

In this paper, we have tried to develop a simple and efficient wavelet-based numerical scheme for solution of fractional differential equations. This technique is based on implementation of Green function along with CAS wavelets. This method is not only easy to implement but also it does not require the conventional operational matrices of integration. The convergence of proposed method has been derived and elaborated in the context of error analysis for further applications. The method has been further analyzed in terms of the efficiency and accuracy by considering a number of documented examples. Moreover, the comparison from these results have also been documented in tabular form against previous studies $[39,40]$.

The present paper is organized as follows: In the second section we review some basic definitions needed in the sequel. We give a brief introduction of CAS wavelet method. Moreover, for the sake of completeness, the CAS wavelet operational matrix is also discussed. In Section 3, we present numerical method called Green-CAS method for fractional boundary value problems. The procedure for implementation of the method is also elaborated in this section. In the fourth section of the paper, we establish sufficient conditions for the existence of unique solution for certain class of boundary value problems with mixed boundary conditions. Section 5 gives comparison of the Green-CAS method with some previous studies $[39,40]$. The method is tested for a number of problems to study the efficiency and accuracy. Furthermore, in Section 6, we workout error analysis for the method. Finally, in last section we conclude the work.

\section{Fractional Calculus and CAS Wavelets}

In this section, we review some basic definitions of fractional calculus. These definitions serve as underpinnings infrastructure for the upcoming section.

Definition 1. [41] The Riemann-Liouville fractional integral operator of order $\alpha \in \mathbb{R}^{+}$is defined as

$$
\mathcal{I}_{t}^{\alpha} f(t)=\frac{1}{\Gamma(\alpha)} \int_{a}^{t}(t-\xi)^{\alpha-1} f(\xi) d \xi,
$$


for $a<t \leq b$.

Definition 2. [41] The Caputo fractional derivative operator of order $\alpha \in \mathbb{R}^{+}$is defined as

$$
\mathcal{D}_{t}^{\alpha} f(t)=\frac{1}{\Gamma(n-\alpha)} \int_{a}^{t}(t-\xi)^{n-\alpha-1} \sigma^{(n)} f(\xi) d \xi,
$$

for $a<t \leq b$, where $\sigma=\frac{d}{d t}$ and $n-1<\alpha \leq n, n \in \mathbb{N}$ and $n=\lfloor\alpha\rfloor+1$.

Lemma 1. [24] Assume that $u_{r+1}(t) \in L^{2}[0,1]$ is a differentiable and bounded function such that there exits $\gamma>0 ; \forall t \in[0,1]:\left|\frac{d^{2}}{d t^{2}} u_{r+1}(t)\right| \leq \gamma$. Let $u_{r+1}^{k, M}(t)$ be the approximation of $u_{r+1}(t)$, then we have

$$
\left|u_{r+1}(t)-u_{r+1}^{k, M}(t)\right| \leq \frac{\gamma}{\pi^{2}} \sum_{n=2^{k}}^{\infty} \sum_{\hat{m}=M+1}^{\infty} \frac{1}{(n+1)^{\frac{5}{2}} \hat{m}^{2}},
$$

and $u_{r+1}^{k, M}(t)$ converges to $u_{r+1}(t)$ as $k, M$ and $r$ approach $\infty$.

Wavelets are special kind of family of functions constructed from specific transformations namely translation and dilation of a single function called the mother wavelet,

$$
\psi_{a, b}(t)=|a|^{-\frac{1}{2}} \psi\left(\frac{t-b}{a}\right), \quad a, b \in \mathbb{R}, a \neq 0 .
$$

If the parameter $|a|<1$, then the wavelet (4) corresponds to higher frequencies with smaller support in time domain and becomes compressed form of mother wavelet. On contrary, when $|a|>1$ the wavelet has larger support in time domain and corresponds to lower frequencies. Discretizing the parameters as $a=a_{0}^{-k}, b=n b_{0} a_{0}^{-k}, a_{0}>1, b_{0}>1$ where $k$ and $n$ are positive integers, we obtain the following family of discrete wavelets

$$
\psi_{k, n}(t)=\left|a_{0}\right|^{\frac{k}{2}} \psi\left(a_{0}^{k} t-n b_{0}\right),
$$

where $\psi_{k, n}(t)$ form a basis for $L^{2}(\mathbb{R})$. In particular, if $a_{0}=2$ and $b_{0}=1$, then these wavelets produce an orthonormal basis. The orthonormal CAS wavelets [20] are defined on the interval $[0,1]$ as

$$
\psi_{k, n}(t)= \begin{cases}2^{\frac{k}{2}} C A S_{\hat{m}}\left(2^{k} t-n+1\right), & \text { for } t \in\left[\frac{n-1}{2^{k}}, \frac{n}{2^{k}}\right] \\ 0, & \text { otherwise; }\end{cases}
$$

where $C A S_{\hat{m}}(t)=\cos (2 \hat{m} \pi t)+\sin (2 \hat{m} \pi t)$ and $n=1,2,3, \cdots, 2^{k}$, is the translation parameter. The non-negative integer $k$ is the level of resolution and $\hat{m}$ is any positive integer. CAS wavelet have compact support, i.e.,

$$
\operatorname{supp}\left(\psi_{k, n}(t)\right)=\overline{\left\{t: \psi_{k, n}(t) \neq 0\right\}}=\left[\frac{n-1}{2^{k}}, \frac{n}{2^{k}}\right] .
$$

\subsection{Function Approximations}

Any function $u(t) \in L^{2}[0,1)$ can be expressed as CAS wavelet series:

$$
u(t)=\sum_{n=0}^{\infty} \sum_{\hat{m} \in \mathbf{Z}} c_{n \hat{m}} \psi_{n, \hat{m}}(t),
$$


where $c_{n \hat{m}}=<u(t), \psi_{n, \hat{m}}(t)>=\int_{0}^{1} u(t) \psi_{n, \hat{m}}(t) d t$. Therefore, the series (8) is truncated as

$$
u(t) \cong \sum_{n=0}^{2^{k}-1} \sum_{\hat{m}=-M}^{M} c_{n \hat{m}} \psi_{n, \hat{m}}(t)=C^{T} \Psi(t)
$$

where $C$ and $\Psi$ are vectors of the form

$$
\begin{aligned}
C= & {\left[c_{0,-M}, c_{0,-M+1}, \cdots, c_{0, M}, c_{1,-M}, c_{1,-M+1}, \cdots, c_{1, M}, \cdots, c_{2^{k}-1,-M^{\prime}}, c_{2^{k}-1,-M+1}, \cdots, c_{2^{k}-1, M}\right]^{T}, } \\
\Psi(t)= & {\left[\psi_{0,-M}(t), \psi_{0,-M+1}(t), \cdots, \psi_{0, M}(t), \psi_{1,-M}(t), \psi_{1,-M+1}(t), \cdots, \psi_{1, M}(t), \cdots, \psi_{2^{k}-1,-M}(t),\right.} \\
& \left.\psi_{2^{k}-1,-M+1}(t), \cdots, \psi_{2^{k}-1, M}(t)\right]^{T} .
\end{aligned}
$$

The collocation points for the CAS wavelet are taken as $t_{i}=\frac{2 i-1}{2 m}$, where $i=1,2, \cdots, m$. The CAS wavelet matrix $\Psi_{m, m}$ can be written as

$$
\Psi_{m \times m}=\left[\Psi\left(\frac{1}{2 m}\right), \Psi\left(\frac{3}{2 m}\right), \cdots, \Psi\left(\frac{2 m-1}{2 m}\right)\right] .
$$

If we fix $k=1, M=1$, we have $n=0,1 ; \hat{m}=-1,0,1$ and $i=1,2, \cdots, 6$, then the CAS wavelet matrix is given by

$$
\Psi_{6 \times 6}=\left(\begin{array}{cccccc}
-0.5176 & -1.4142 & 1.9319 & 0 & 0 & 0 \\
0 & 0 & 0 & -0.5176 & -1.4142 & 1.9319 \\
1.4142 & 1.4142 & 1.4142 & 0 & 0 & 0 \\
0 & 0 & 0 & 1.4142 & 1.4142 & 1.4142 \\
1.9319 & -1.4142 & -0.5176 & 0 & 0 & 0 \\
0 & 0 & 0 & 1.9319 & -1.4142 & -0.5176
\end{array}\right) .
$$

\subsection{CAS Wavelet Operational Matrix}

For simplicity Equation (9) can be written as

$$
u(t) \cong \sum_{i=1}^{m} c_{i} \psi_{i}(t)=C^{T} \Psi(t)
$$

where the index $i$ is determined by the expression $i=M(2 n+1)+(\hat{m}+n+1)$ and $m=2^{k}(2 M+1)$.

Any arbitrary function $u(t) \in L^{2}(\mathbb{R})$ can be expanded in terms of a block-pulse function [42] given as

$$
u(t) \cong \sum_{i=1}^{m} a_{i} b_{i}(t)=A^{T} \mathbf{B}(t)
$$

the term $a_{i}$ is the coefficient of the block-pulse functions $b_{i}$. So the CAS wavelet can be expanded in terms of block-pulse as

$$
\Psi(t)=\Psi_{m \times m} \mathbf{B}(t) .
$$

The fractional integral of block-pulse function is given by

$$
\left(\mathcal{I}_{t}^{\alpha} \mathbf{b}\right)(t)=K_{m \times m}^{\alpha} \mathbf{B}(t),
$$

where $K_{m \times m}^{\alpha}$ is given in [18] with $P_{m \times m}^{\alpha}=\Psi_{m \times m} K_{m \times m}^{\alpha}\left(\Psi_{m \times m}\right)^{-1}$. The operational matrix $P_{m \times m}^{\alpha}$ for $\alpha=0.75$ and for fixed values of $k=1$ and $M=1$ is given by 


$$
P_{6 \times 6}^{0.75}=\left(\begin{array}{cccccc}
0.0584 & -0.0087 & -0.1227 & 0.0367 & 0.0620 & 0.0166 \\
0 & 0.0584 & 0 & -0.1227 & 0 & 0.0620 \\
0.1091 & -0.0258 & 0.3697 & 0.5041 & -0.1227 & 0.0367 \\
0 & 0.1091 & 0 & 0.3697 & 0 & -0.1227 \\
-0.0893 & 0.0052 & 0.1091 & -0.0258 & 0.0584 & -0.0087 \\
0 & -0.0893 & 0 & 0.1091 & 0 & 0.0584
\end{array}\right)
$$

\section{Approximate Solutions of BVP via Green-CAS Wavelet Method}

We present the implementation procedure for the proposed method for solving linear and non-linear ordinary fractional boundary value problems numerically, called Green-CAS technique. In general, this method does not require the use of operational matrix for fractional differential equation. Interestingly, accuracy is not compromised, rather enhanced by using Green-CAS method for solving fractional boundary value problems.

Consider the following class of fractional boundary value problem:

$$
\mathcal{D}_{0}^{\alpha} u(t)+f(t, u(t))=0, \quad \text { with } u(0)=u_{0}, \quad u(1)=u_{1} .
$$

Lemma 2. [43] Let $0<\alpha \leq 2$, and $f(t, u(t)):[0,1] \times \mathbb{R} \longrightarrow \mathbb{R}$ is continuous, then $u(t)$ is the solution of Equation (17) if and only if $u(t)$ satisfies the following Fredholm integral equation

$$
u(t)=\int_{0}^{1} G(t, \xi) f(\xi, u(\xi)) d \xi+u_{0}+t\left(u_{1}-u_{0}\right),
$$

where

$$
G(t, \xi)= \begin{cases}\frac{1}{\Gamma(\alpha)}\left[-(t-\xi)^{\alpha-1}+t(1-\xi)^{\alpha-1}\right], & \text { if } 0 \leq \xi<t \\ \frac{t}{\Gamma(\alpha)}(1-\xi)^{\alpha-1}, & \text { if } t \leq \xi \leq 1 .\end{cases}
$$

The graph for the function in Equation (19), for $\alpha=2, k=3$, and $M=5$, is shown in Figure 1 .

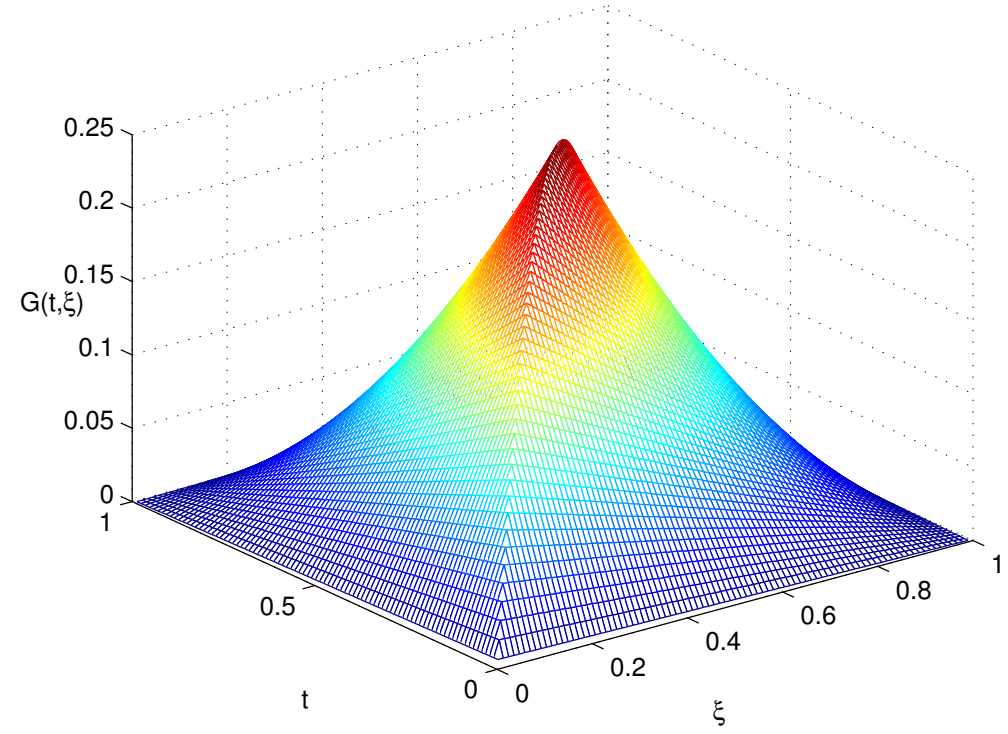

Figure 1. Green function for fixed values of $M=5, k=3$ and for $\alpha=2$. 
Numerical Procedure to Fractional Linear Differential Equations

In this subsection, we describe the procedure of implementation of Green-CAS method to approximate the numerical solutions for the linear fractional differential equations with boundary conditions.

Consider the class of linear fractional differential equations

$$
\mathcal{D}_{t}^{\alpha} u(t)+a(t) \mathcal{D}_{t}^{\beta} u(t)+b(t) u^{\prime}(t)+d(t) u(t)=g(t), \quad 1<\alpha \leq 2,0<\beta \leq 1,
$$

with Dirichlet boundary condition

$$
u(0)=p_{1}, \quad u(1)=p_{2},
$$

or mixed boundary conditions of the form

$$
\text { (1) } u^{\prime}(0)=q_{1}, u(1)=q_{2} \text { or }(2) \quad u(0)=r_{1}, \quad u^{\prime}(1)=r_{2}
$$

where $t \in[0,1]$ and $p_{1}, p_{2}, q_{1}, q_{2}, r_{1}$ and $r_{2}$ are constants and $g$ is a linear function.

Case \#1 Equation (20) with Dirichlet boundary condition (21). Approximate the higher order derivative term as

$$
\mathcal{D}_{t}^{\alpha} u(t)=\sum_{n=0}^{2^{k}-1} \sum_{\hat{m}=-M}^{M} c_{n \hat{m}} \psi_{n, \hat{m}}(t) .
$$

Applying the fractional integral operator on both sides of Equation (23), we get

$$
u(t)=\sum_{n=0}^{2^{k}-1} \sum_{\hat{m}=-M}^{M} c_{n \hat{m}}\left(\mathcal{I}_{t}^{\alpha} \psi_{n, \hat{m}}(t)\right)+t y_{1}+y_{2} .
$$

Using the boundary conditions (21), Equation (24) becomes

$$
\begin{gathered}
u(t)=\sum_{n=0}^{2^{k}-1} \sum_{\hat{m}=-M}^{M} c_{n \hat{m}}\left(\mathcal{I}_{t}^{\alpha} \psi_{n, \hat{m}}(t)\right)+t\left(p_{2}-p_{1}-\sum_{n=0}^{2^{k}-1} \sum_{\hat{m}=-M}^{M} c_{n \hat{m}}\left(\mathcal{I}_{t}^{\alpha} \psi_{n, \hat{m}}(1)\right)\right)+p_{1} . \\
\left.\left.u(t)=\sum_{n=0}^{2^{k}-1} \sum_{\hat{m}=-M}^{M} c_{n \hat{m}}\left(\int_{0}^{t} \frac{(t-\xi)^{\alpha-1}}{\Gamma(\alpha)} \psi_{n, \hat{m}}(\xi)\right) d \xi-t \int_{0}^{1} \frac{(1-\xi)^{\alpha-1}}{\Gamma(\alpha)} \psi_{n, \hat{m}}(\xi)\right) d \xi\right)+t\left(p_{2}-p_{1}\right)+p_{1} \\
\left.u(t)=\sum_{n=0}^{2^{k}-1} \sum_{\hat{m}=-M}^{M} c_{n \hat{m}} \int_{0}^{1} G_{1}(t, \xi) \psi_{n, \hat{m}}(\xi)\right) d \xi+t\left(p_{2}-p_{1}\right)+p_{1},
\end{gathered}
$$

where

$$
G_{1}(t, \xi)= \begin{cases}\frac{1}{\Gamma(\alpha)}\left((t-\xi)^{\alpha-1}-t(1-\xi)^{\alpha-1}\right), & \text { if } 0 \leq \xi<t \\ -\frac{t}{\Gamma(\alpha)}(1-\xi)^{\alpha-1}, & \text { if } t \leq \xi \leq 1 .\end{cases}
$$

Differentiating both sides of the Equation (25)

$$
\begin{gathered}
u^{\prime}(t)=\sum_{n=0}^{2^{k}-1} \sum_{\hat{m}=-M}^{M} c_{n \hat{m}}\left(\mathcal{I}_{t}^{\alpha-1} \psi_{n, \hat{m}}(t)\right)+\left(p_{2}-p_{1}-\sum_{n=0}^{2^{k}-1} \sum_{\hat{m}=-M}^{M} c_{n \hat{m}}\left(\mathcal{I}_{t}^{\alpha} \psi_{n, \hat{m}}(1)\right)\right) . \\
\left.\left.u^{\prime}(t)=\sum_{n=0}^{2^{k}-1} \sum_{\hat{m}=-M}^{M} c_{n \hat{m}}\left(\int_{0}^{t} \frac{(t-\xi)^{\alpha-2}}{\Gamma(\alpha-1)} \psi_{n, \hat{m}}(\xi)\right) d \xi-\int_{0}^{1} \frac{(1-\xi)^{\alpha-1}}{\Gamma(\alpha)} \psi_{n, \hat{m}}(\xi)\right) d \xi\right)+p_{2}-p_{1}
\end{gathered}
$$




$$
\left.u^{\prime}(t)=\sum_{n=0}^{2^{k}-1} \sum_{\hat{m}=-M}^{M} c_{n \hat{m}} \int_{0}^{1} G_{2}(t, \xi) \psi_{n, \hat{m}}(\xi)\right) d \xi+\left(p_{2}-p_{1}\right)
$$

where

$$
G_{2}(t, \xi)= \begin{cases}\frac{1}{\Gamma(\alpha-1)}(t-\xi)^{\alpha-2}-\frac{1}{\Gamma(\alpha)}(1-\xi)^{\alpha-1}, & \text { if } 0 \leq \xi<t \\ -\frac{1}{\Gamma(\alpha)}(1-\xi)^{\alpha-1}, & \text { if } t \leq \xi \leq 1\end{cases}
$$

Similarly taking $\beta$ order derivative on both sides in Equation (25)

$$
\begin{gathered}
\mathcal{D}_{t}^{\beta} u(t)=\sum_{n=0}^{2^{k}-1} \sum_{\hat{m}=-M}^{M} c_{n \hat{m}}\left(\mathcal{I}_{t}^{\alpha-\beta} \psi_{n, \hat{m}}(t)\right)+\frac{t^{1-\beta}}{\Gamma(2-\beta)}\left(p_{2}-p_{1}-\sum_{n=0}^{2^{k}-1} \sum_{\hat{m}=-M}^{M} c_{n \hat{m}}\left(\mathcal{I}_{t}^{\alpha} \psi_{n, \hat{m}}(1)\right)\right) \\
\left.\left.\mathcal{D}_{t}^{\beta} u(t)=\sum_{n=0}^{2^{k}-1} \sum_{\hat{m}=-M}^{M} c_{n \hat{m}}\left(\int_{0}^{t} \frac{(t-\xi)^{\alpha-\beta-1}}{\Gamma(\alpha-\beta)} \psi_{n, \hat{m}}(\xi)\right) d \xi-\int_{0}^{1} \frac{t^{1-\beta}}{\Gamma(2-\beta)} \frac{(1-\xi)^{\alpha-1}}{\Gamma(\alpha)} \psi_{n, \hat{m}}(\xi)\right) d \xi\right)+\frac{t^{1-\beta}}{\Gamma(2-\beta)}\left(p_{2}-p_{1}\right) \\
\left.\mathcal{D}_{t}^{\beta} u(t)=\sum_{n=0}^{2^{k}-1} \sum_{\hat{m}=-M}^{M} c_{n \hat{m}} \int_{0}^{1} G_{3}(t, \xi) \psi_{n, \hat{m}}(\xi)\right) d \xi+\frac{t^{1-\beta}}{\Gamma(2-\beta)}\left(p_{2}-p_{1}\right)
\end{gathered}
$$

where

$$
G_{3}(t, \xi)= \begin{cases}\frac{1}{\Gamma(\alpha-\beta)}(t-\xi)^{\alpha-\beta-1}-\frac{t^{1-\beta}}{\Gamma(2-\beta) \Gamma(\alpha)}(1-\xi)^{\alpha-1}, & \text { if } 0 \leq \xi<t \\ -\frac{t^{1-\beta}}{\Gamma(2-\beta) \Gamma(\alpha)}(1-\xi)^{\alpha-1}, & \text { if } t \leq \xi \leq 1\end{cases}
$$

Inserting (23), (26), (28) and (30) into (20), we obtain

$$
\sum_{n=0}^{2^{k}-1} \sum_{\hat{m}=-M}^{M} c_{n \hat{m}}\left(\psi_{n, \hat{m}}(\xi)+a(t) \int_{0}^{1} G_{3}(t, \xi) \psi_{n, \hat{m}}(\xi) d \xi+b(t) \int_{0}^{1} G_{2}(t, \xi) \psi_{n, \hat{m}}(\xi) d \xi+d(t) \int_{0}^{1} G_{1}(t, \xi) \psi_{n, \hat{m}}(\xi) d \xi\right)=f(t),
$$

where $f(t)=-\left(p_{2}-p_{1}\right)\left[a(t) \frac{t^{1-\beta}}{\Gamma(2-\beta)}+b(t)+t d(t)\right]+d(t) p_{1}+g(t)$.

The Equation (31) at the collocation points $t_{i}=\frac{2 i-1}{2 m}, i=1,2,3, \cdots, m$, in matrix form is

$$
C^{T}\left\{\Psi_{m \times m}+A \hat{G}_{3 m \times m} \Psi_{m \times m}+B \hat{G}_{2 m \times m} \Psi_{m \times m}+D \hat{G}_{1 m \times m} \Psi_{m \times m}\right\}=F^{T} .
$$

Case \#2(a) Equation (20) with mixed boundary condition 22 (1), Equation (24) can be written as

$$
\begin{gathered}
u(t)=\sum_{n=0}^{2^{k}-1} \sum_{\hat{m}=-M}^{M} c_{n \hat{m}}\left(\mathcal{I}_{t}^{\alpha} \psi_{n, \hat{m}}(t)\right)+t q_{1}+\left(q_{2}-q_{1}-\sum_{n=0}^{2^{k}-1} \sum_{\hat{m}=-M}^{M} c_{n \hat{m}}\left(\mathcal{I}_{x}^{\alpha} \psi_{n, \hat{m}}(1)\right)\right) . \\
\left.u(t)=\sum_{n=0}^{2^{k}-1} \sum_{\hat{m}=-M}^{M} c_{n \hat{m}} \int_{0}^{1} G_{4}(t, \xi) \psi_{n, \hat{m}}(\xi)\right) d \xi+t q_{1}+q_{2}-q_{1},
\end{gathered}
$$

where

$$
G_{4}(t, \xi)= \begin{cases}\frac{1}{\Gamma(\alpha)}\left((t-\xi)^{\alpha-1}-(1-\xi)^{\alpha-1}\right), & \text { if } 0 \leq \xi<t \\ -\frac{1}{\Gamma(\alpha)}(1-\xi)^{\alpha-1}, & \text { if } t \leq \xi \leq 1\end{cases}
$$

Again, the first order and $\beta$ order derivative of $u$ in Equation (33) has the form

$$
\begin{gathered}
u^{\prime}(t)=\sum_{n=0}^{2^{k}-1} \sum_{\hat{m}=-M}^{M} c_{n \hat{m}}\left(\mathcal{I}_{t}^{\alpha-1} \psi_{n, \hat{m}}(t)\right)+q_{1}=\sum_{n=0}^{2^{k}-1} \sum_{\hat{m}=-M}^{M} c_{n \hat{m}} P_{1}^{\alpha-1} \psi_{n, \hat{m}}(t)+q_{1}, \\
\mathcal{D}_{t}^{\beta} u(t)=\sum_{n=0}^{2^{k}-1} \sum_{\hat{m}=-M}^{M} c_{n \hat{m}}\left(\mathcal{I}_{t}^{\alpha-\beta} \psi_{n, \hat{m}}(t)\right)+\frac{t^{1-\beta}}{\Gamma(2-\beta)} q_{1}=\sum_{n=0}^{2^{k}-1} \sum_{\hat{m}=-M}^{M} c_{n \hat{m}} P_{2}^{\alpha-\beta} \psi_{n, \hat{m}}(t)+\frac{t^{1-\beta}}{\Gamma(2-\beta)} q_{1} .
\end{gathered}
$$


Substituting equations (23) and (34)-(36) into Equation (20), we have

$$
C^{T}\left\{\Psi_{m \times m}+A P_{1}^{\alpha-1} \Psi_{m \times m}+B P_{2}^{\alpha-\beta} \Psi_{m \times m}+D \hat{G}_{4 m \times m} \Psi_{m \times m}\right\}=K^{T},
$$

where $K^{T}$ is known vector that is the approximation of function $k(t)=-q_{1}\left[a(t) \frac{t^{1-\beta}}{\Gamma(2-\beta)}+b(t)+d(t)(t-\right.$ $1)]-d(t) q_{2}+g(t)$.

Case \#2(b) Equation (20) with mixed boundary condition 22 (2), the Equation (24) becomes

$$
\begin{gathered}
u(t)=\sum_{n=0}^{2^{k}-1} \sum_{\hat{m}=-M}^{M} c_{n \hat{m}}\left(\mathcal{I}_{t}^{\alpha} \psi_{n, \hat{m}}(t)\right)+t\left(r_{2}-\sum_{n=0}^{2^{k}-1} \sum_{\hat{m}=-M}^{M} c_{n \hat{m}}\left(\mathcal{I}_{x}^{\alpha-1} \psi_{n, \hat{m}}(1)\right)\right)+r_{1} . \\
\left.u(t)=\sum_{n=0}^{2^{k}-1} \sum_{\hat{m}=-M}^{M} c_{n \hat{m}} \int_{0}^{1} G_{5}(t, \xi) \psi_{n, \hat{m}}(\xi)\right) d s d \xi+t r_{2}+r_{1},
\end{gathered}
$$

where

$$
G_{5}(t, \xi)= \begin{cases}\frac{1}{\Gamma(\alpha)}(t-\xi)^{\alpha-1}-\frac{t}{\Gamma(\alpha-1)}(1-\xi)^{\alpha-2}, & \text { if } 0 \leq \xi<t \\ -\frac{t}{\Gamma(\alpha-1)}(1-\xi)^{\alpha-2}, & \text { if } t \leq \xi \leq 1\end{cases}
$$

Again, the first order and $\beta$-order derivative of $u$ in Equation (38) has the form

$$
\begin{gathered}
\left.\left.u^{\prime}(t)=\sum_{n=0}^{2^{k}-1} \sum_{\hat{m}=-M}^{M} c_{n \hat{m}}\left(\int_{0}^{t} \frac{(t-\xi)^{\alpha-2}}{\Gamma(\alpha-1)} \psi_{n, \hat{m}}(\xi)\right) d \xi-\int_{0}^{1} \frac{(1-\xi)^{\alpha-2}}{\Gamma(\alpha-1)} \psi_{n, \hat{m}}(\xi)\right) d \xi\right)+r_{2}, \\
\left.u^{\prime}(t)=\sum_{n=0}^{2^{k}-1} \sum_{\hat{m}=-M}^{M} c_{n \hat{m}} \int_{0}^{1} G_{6}(t, \xi) \psi_{n, \hat{m}}(\xi)\right) d \xi+r_{2}
\end{gathered}
$$

where

$$
G_{6}(t, \xi)= \begin{cases}\frac{1}{\Gamma(\alpha-1)}\left\{(t-\xi)^{\alpha-2}-(1-\xi)^{\alpha-2}\right\}, & \text { if } 0 \leq \xi<t \\ -\frac{1}{\Gamma(\alpha-1)}(1-\xi)^{\alpha-2}, & \text { if } t \leq \xi \leq 1\end{cases}
$$

and

$$
\begin{gathered}
\left.\left.\mathcal{D}_{t}^{\beta} u(t)=\sum_{n=0}^{2^{k}-1} \sum_{\hat{m}=-M}^{M} c_{n \hat{m}}\left(\int_{0}^{t} \frac{(t-\xi)^{\alpha-\beta-1}}{\Gamma(\alpha-\beta)} \psi_{n, \hat{m}}(\xi)\right) d \xi-\frac{t^{1-\beta}}{\Gamma(2-\beta)} \int_{0}^{1} \frac{(1-\xi)^{\alpha-2}}{\Gamma(\alpha-1)} \psi_{n, \hat{m}}(\xi)\right) d \xi\right)+\frac{t^{1-\beta}}{\Gamma(2-\beta)} r_{2} \\
\left.\mathcal{D}_{t}^{\beta} u(t)=\sum_{n=0}^{2^{k}-1} \sum_{\hat{m}=-M}^{M} c_{n \hat{m}} \int_{0}^{1} G_{7}(t, \xi) \psi_{n, \hat{m}}(\xi)\right) d \xi+\frac{t^{1-\beta}}{\Gamma(2-\beta)} r_{2}
\end{gathered}
$$

where

$$
G_{7}(t, \xi)= \begin{cases}\frac{1}{\Gamma(\alpha-\beta)}(t-\xi)^{\alpha-\beta-1}-\frac{t^{1-\beta}}{\Gamma(\alpha-1) \Gamma(2-\beta)}(1-\xi)^{\alpha-2}, & \text { if } 0 \leq \xi<t \\ -\frac{t^{1-\beta}}{\Gamma(2-\beta) \Gamma(\alpha-1)}(1-\xi)^{\alpha-2}, & \text { if } t \leq \xi \leq 1\end{cases}
$$

Using (23) and (39)-(41) in (20), we obtain

$$
\sum_{n=0}^{2^{k}-1} \sum_{\hat{m}=-M}^{M} c_{n \hat{m}}\left(\psi_{n, \hat{m}}(\xi)+a(t) \int_{0}^{1} G_{7}(t, \xi) \psi_{n, \hat{m}}(\xi) d \xi+b(t) \int_{0}^{1} G_{6}(t, \xi) \psi_{n, \hat{m}}(\xi) d \xi+d(t) \int_{0}^{1} G_{5}(t, \xi) \psi_{n, \hat{m}}(\xi) d \xi\right)=s(t),
$$

where $s(t)=-r_{2}\left[a(t) \frac{t^{1-\beta}}{\Gamma(2-\beta)}+b(t)+t d(t)\right]-d(t) r_{1}+g(t)$. 
The Equation (42) can be written in vector form and in matrix notation once the collocation points $t_{i}=\frac{2 i-1}{2 m}, i=1,2,3, \cdots, m$, are used

$$
C^{T}\left\{\Psi_{m \times m}+A \hat{G}_{7 m \times m} \Psi_{m \times m}+B \hat{G}_{6 m \times m} \Psi_{m \times m}+D \hat{G}_{5 m \times m} \Psi_{m \times m}\right\}=S^{T} .
$$

For the sake of convenience, we use some matrix notations.

The $\hat{G}_{1}, \hat{G}_{2}, \cdots, \hat{G}_{7}$, can be obtain by using CAS wavelet approximations and orthogonality property of wavelets. For example, the Green function can be approximated by CAS wavelet as follows

$$
G_{1}(t, \xi)=\sum_{n=0}^{2^{k}-1} \sum_{\hat{m}=-M}^{M}\left[\psi_{n, \hat{m}}(t)\right]^{T} \hat{G}_{1} \psi_{n, \hat{m}}(\xi)
$$

Therefore

$$
\sum_{n=0}^{2^{k}-1} \sum_{\hat{m}=-M}^{M} \int_{0}^{1} G_{1}(t, \xi) \psi_{n, \hat{m}}(\xi) d \xi=\hat{G}_{1 m \times m} \Psi_{m \times m}
$$

By using the orthogonality of the sequence $\left\{\psi_{n, m}(t)\right\}$ on $[0,1]$, we get

$$
\sum_{n=0}^{2^{k}-1} \sum_{\hat{m}=-M}^{M} \int_{0}^{1} \psi_{n, m}(\xi)\left[\psi_{n, m}(\xi)\right]^{T} d \xi=\mathbb{I}_{m \times m}
$$

where $\mathbb{I}_{m \times m}$ is an identity matrix with dimension $m \times m$.

Similarly,

$$
\sum_{n=0}^{2^{k}-1} \sum_{\hat{m}=-M}^{M} \int_{0}^{1} G_{j}(t, \xi) \psi_{n, \hat{m}}(\xi) d \xi=\hat{G}_{j_{m \times m}} \Psi_{m \times m}, \quad \text { for } j=1,2, \ldots, 7,
$$

where $C=\left\{C_{1}, C_{2}, C_{3}, \cdots, C_{m}\right\}$ is an unknown vector, and $A, B, D$ are the diagonal matrices given by

$$
A=\left[\begin{array}{cccc}
a\left(t_{1}\right) & 0 & \cdots & 0 \\
0 & a\left(t_{2}\right) & \cdots & 0 \\
\vdots & \vdots & \ddots & \vdots \\
0 & 0 & \cdots & a\left(t_{m}\right)
\end{array}\right], B=\left[\begin{array}{cccc}
b\left(t_{1}\right) & 0 & \cdots & 0 \\
0 & b\left(t_{2}\right) & \cdots & 0 \\
\vdots & \vdots & \ddots & \vdots \\
0 & 0 & \cdots & b\left(t_{m}\right)
\end{array}\right] \text { and } D=\left[\begin{array}{cccc}
d\left(t_{1}\right) & 0 & \cdots & 0 \\
0 & d\left(t_{2}\right) & \cdots & 0 \\
\vdots & \vdots & \ddots & \vdots \\
0 & 0 & \cdots & d\left(t_{m}\right)
\end{array}\right]
$$

The Green-CAS wavelet coefficient vector $C$ can be obtained from the algebraic equations (32) and (37) or (32) and (43). This in turn gives the approximate solution when inserted in (26), (34) and (39) respectively.

\section{Existence and Uniqueness}

The primary objective of this paper is to develop a numerical scheme for fractional boundary value problems. In practice, however, one may be typically interested in finding the conditions for the existence of solutions on given domain. We know establish the conditions under which there exists unique solution of fractional boundary value problems. There is a vast literature on the existence theory of fractional boundary value problems. We refer the interested readers to [44-47] and references therein. We examine the existence and uniqueness of solution for the class of fractional differential equations (20) subject to relatively general mixed boundary conditions

$$
\mu_{1} u(0)+\mu_{2} u^{\prime}(0)=p, \quad \eta_{1} u(1)+\eta_{2} u^{\prime}(1)=q,
$$

where $\mu_{1}, \mu_{2}, \eta_{1}, \eta_{2}, p, q \in \mathbb{R}$. Please note that for $\mu_{1}=1, \mu_{2}=0$, and $p=p_{1}$, and $\eta_{1}=1, \eta_{2}=0, q=p_{2}$, the boundary conditions (45) reduce to boundary condition in (21). Furthermore, for $\mu_{1}=0, \mu_{2}=1$, 
and $p=q_{1}$, and $\eta_{1}=1, \eta_{2}=0, q=q_{2}$, the boundary conditions (45) reduce to boundary the first condition in (22). Finally, for $\mu_{1}=1, \mu_{2}=0$, and $p=r_{1}$, and $\eta_{1}=0, \eta_{2}=1, q=r_{2}$, the boundary conditions (45) reduces to the second boundary condition in (22).

For convenience, we introduce following notations:

$g(t, u, v, w):=f(t)-a(t) v(t)-b(t) w(t)-d(t) u(t)$, where $v(t):=D^{\beta} u(t)$ and $w(t):=u^{\prime}(t)$. Also, we define $\lambda(t)=\frac{1}{\Delta}\left(\mu_{2}-t \mu_{1}\right)$, where $\Delta=\mu_{1}\left(\eta_{1}+\eta_{2}\right)-\eta_{1} \mu_{2}$.

In the following, we transform the boundary value problem (20) and (45) to an equivalent integral equation.

Lemma 3. Assume $a, b, d$ and $f$ are continuous real valued functions defined on $[0,1]$ and $\Delta \neq 0$. Then $u \in C[0,1]$ is a solution of the boundary value problem (20) and (45) iff $u$ solves the integral equation

$$
u(t)=\int_{0}^{1} \mathcal{G}(t, s) g(s, u(s), v(s), w(s)) d s,
$$

where

$$
\mathcal{G}(t, s)= \begin{cases}\frac{1}{\Gamma(\alpha)}\left[(t-s)^{\alpha-1}+\eta_{1} \lambda(t)(1-s)^{\alpha-1}\right]+\frac{\eta_{2} \lambda(t)}{\Gamma(\alpha-1)}(1-s)^{\alpha-2}, & \text { for } s \leq t \\ \frac{\eta_{1} \lambda(t)}{\Gamma(\alpha)}(1-s)^{\alpha-1}+\frac{\eta_{2} \lambda(t)}{\Gamma(\alpha-1)}(1-s)^{\alpha-2}, & \text { for } s \leq t\end{cases}
$$

We define the set $\mathcal{B}=\left\{u(t): D_{t}^{\varrho} u \in C[0,1]\right\}$, where $\varrho \in\{0, \beta, 1\}$ and norm for this set as $\|u\|=\sum_{\varrho} \max _{t \in[0,1]}\left|D^{\varrho} u(t)\right|$. The space $\mathcal{B}$ furnished with the norm $\|$.$\| is a Banach space.$

The proof of Lemma 3 is similar to the proof of Lemma 2.1 in [45].

Theorem 1. Under the assumptions of Lemma 3, there exists at least one solution of the boundary value problem (20) and (45), provided $L(B+C+\tilde{D})<\Gamma(\alpha)$.

Proof. The fixed points of the operator $\Psi: \mathcal{B} \rightarrow \mathcal{B}$ defined as

$$
\Psi u(t)=\int_{0}^{1} \mathcal{G}(t, s) g(s, u(s), v(s), w(s)) d s,
$$

are the solutions of the boundary value problem (20) and (45). Let $\tilde{f}, \tilde{a}, \tilde{b}, \tilde{d}$ and $\tilde{\lambda}$ be maximum values for modulus of $f, a, b, d$ and $\lambda$ respectively and $L:=\max \{\tilde{a}, \tilde{b}, \tilde{d}\}$. Define the set $\Omega=\{u \in \mathcal{B}:\|u\| \leq$ $\mathcal{R}\}$, where $\mathcal{R}$ is chosen so that it satisfies the condition $\mathcal{R} \geq \max \left\{\frac{3 B \tilde{f}}{\Gamma(\alpha)-3 A B}, \frac{3 C \tilde{f}}{\Gamma(\alpha)-3 A C}, \frac{3 \tilde{D} \tilde{f}}{\Gamma(\alpha)-3 A \tilde{D}}\right\}$, where $A:=\tilde{a}+\tilde{b}+\tilde{d})$ and $B:=1+\left(\left|\eta_{1}\right|+\left|\eta_{2}\right|(\alpha-1)\right) \tilde{\lambda}, C:=\left(\alpha+\left(\left|\eta_{1}\right|+\left|\eta_{2}\right|\right) \frac{\left|\mu_{1}\right|}{|\Delta|}\right)$ and $\tilde{D}:=$ $\frac{\Gamma(\alpha)}{\Gamma(\alpha-\beta)}+\frac{\left|\eta_{1}\right|+\alpha\left|\eta_{2}\right|}{|\Delta| \Gamma(2-\beta)}$. It is to be noted that

$$
|\Psi u(t)| \leq(\tilde{f}+\mathcal{R} A) \int_{0}^{1}|\mathcal{G}(t, s)| d s .
$$

Since $(t-s)^{\alpha-1} \leq(1-s)^{\alpha-1}$. Therefore, from Equation (47), we have following estimate for $\mathcal{G}$ :

$$
|\mathcal{G}(t, s)| \leq \frac{(1-s)^{\alpha-1}}{\Gamma(\alpha)}\left(1+\left|\eta_{1}\right| \tilde{\lambda}\right)+\frac{\left|\eta_{1}\right| \tilde{\lambda}}{\Gamma(\alpha-1)}(1-s)^{\alpha-2} \leq B \frac{(1-s)^{\alpha-2}}{\Gamma(\alpha-1)} .
$$

Similarly, we have

$$
\left|\frac{d}{d t} \mathcal{G}(t, s)\right| \leq\left(\alpha+\left(\left|\eta_{1}\right|+\left|\eta_{2}\right|\right) \frac{\left|\mu_{1}\right|}{|\Delta|}\right) \frac{(1-s)^{\alpha-2}}{\Gamma(\alpha-1)}=C \frac{(1-s)^{\alpha-2}}{\Gamma(\alpha-1)},\left|D_{t}^{\beta} \mathcal{G}(t, s)\right| \leq \tilde{D} \frac{(1-s)^{\alpha-2}}{\Gamma(\alpha-1)}
$$


Therefore, inequality (49) may further be refined as $\left.|\Psi u(t)| \leq \frac{1}{\Gamma(\alpha)}(\tilde{f}+\mathcal{R} A) B\right) \leq \frac{\mathcal{R}}{3}$. By similar argument, we have the estimates $\left.\left|\Psi^{\prime} u(t)\right| \leq \frac{1}{\Gamma(\alpha)}(\tilde{f}+\mathcal{R} A) C\right) \leq \frac{\mathcal{R}}{3}$, and $\left|D_{t}^{\beta} \Psi u(t)\right| \leq \frac{1}{\Gamma(\alpha)}(\tilde{f}+$ $\mathcal{R} A) \tilde{D}) \leq \frac{\mathcal{R}}{3}$. Therefore $\|\Psi u\| \leq \frac{\mathcal{R}}{3}+\frac{\mathcal{R}}{3}+\frac{\mathcal{R}}{3}=\mathcal{R}$. Hence $\Psi: \Omega \rightarrow \Omega$. Now, we prove that $\Psi$ is contraction. Let $u, \tilde{u} \in \Omega$. Then, we have

$$
\begin{aligned}
|g(t, u, v, w)-g(t, \tilde{u}, \tilde{v}, \tilde{w})| & \leq \tilde{a}|v-\tilde{v}|+\tilde{b}|w-\tilde{w}|+\tilde{d}|u-\tilde{u}| \\
& \leq L(|v-\tilde{v}|+|w-\tilde{w}|+|u-\tilde{u}|) .
\end{aligned}
$$

Therefore, from (48) and above inequality, we have following estimates

$$
\begin{aligned}
|\Psi u(t)-\Psi \tilde{u}(t)| & \leq \int_{0}^{1}|\mathcal{G}(t, s)||g(s, u, v, w)-g(t, \tilde{u}, \tilde{v}, \tilde{w})| d s \\
& \leq L\|u-\tilde{u}\| \int_{0}^{1}|\mathcal{G}(t, s)| d s \leq \frac{L B}{\Gamma(\alpha)}\|u-\tilde{u}\|
\end{aligned}
$$

Similarly, $|\Psi u(t)-\Psi \tilde{u}(t)| \leq \frac{L C}{\Gamma(\alpha)}\|u-\tilde{u}\|$ and $|\Psi u(t)-\Psi \tilde{u}(t)| \leq \frac{L \tilde{D}}{\Gamma(\alpha)}\|u-\tilde{u}\|$.

Finally, $\|\Psi u-\Psi \tilde{u}\| \leq \kappa\|u-\tilde{u}\|$ where $\kappa<1$. Thus, $\Psi$ is contraction. By contraction mapping theorem, there exists a unique solution of the boundary value problem (20) and (45).

Remark 1. Theorem 1 can be proved under weaker conditions on $a, b, d$ and $f$. That is the continuity condition may be dropped and assuming $a, b, d$ and $f$ from the space $L_{1}[0,1]$.

\section{Applications}

In this section, we discuss some numerical examples to test the accuracy of the proposed Green-CAS method. The results are compared with some other numerical methods available in literature.

\subsection{Linear Case}

We implement the Green-CAS wavelet to solve the linear fractional differential equations with boundary conditions.

Example 1. Consider

$$
\mathcal{D}_{t}^{\alpha} u(t)+a(t) u(t)=g(t), \quad 1<\alpha \leq 2, t \in[0,1],
$$

with boundary conditions

$$
u(0)=0, \quad u(1)=0 .
$$

If $a(t)=\sin (t) \cos (t)$ and $g(t)=\frac{\Gamma(\alpha+2)}{\Gamma(3)} t-\frac{\Gamma(3)}{\Gamma(3-\alpha)} t^{2-\alpha}+\sin (t) \cos (t)\left(t^{\alpha}-t\right) t$, then one can find the analytic solution of the above system as $u(t)=t\left(t^{\alpha}-t\right)$. The numerical solutions are obtained by the proposed method discussed in the above section. The absolute error between exact and numerical solutions for the different values of $\alpha, k$ and $M$ are shown in Table 1. The numerical solutions obtained from Green-CAS method are documented in a graphical form in Figure 2 for different values of $\alpha$. The maximum absolute error decrease by increasing value of $M$ and $k$. 
Table 1. Maximum absolute error for different values of $\alpha, k$ and $M$

\begin{tabular}{ccccccc}
\hline & \multicolumn{5}{c}{$k=\mathbf{3}$} & $\boldsymbol{k}=\mathbf{5}$ \\
\cline { 2 - 7 } & $\boldsymbol{M}=\mathbf{3}$ & $\boldsymbol{M}=\mathbf{5}$ & $\boldsymbol{M}=\mathbf{7}$ & $\boldsymbol{M}=\mathbf{3}$ & $\boldsymbol{M}=\mathbf{5}$ & $\boldsymbol{M}=\mathbf{7}$ \\
\hline 1.1 & $6.40456 \times 10^{-4}$ & $3.93807 \times 10^{-4}$ & $2.81392 \times 10^{-4}$ & $1.42542 \times 10^{-4}$ & $8.69363 \times 10^{-5}$ & $6.18848 \times 10^{-5}$ \\
1.3 & $9.77420 \times 10^{-4}$ & $5.47839 \times 10^{-4}$ & $3.67537 \times 10^{-4}$ & $1.64122 \times 10^{-4}$ & $9.13982 \times 10^{-5}$ & $6.11330 \times 10^{-5}$ \\
1.5 & $7.58102 \times 10^{-4}$ & $3.87391 \times 10^{-4}$ & $2.44023 \times 10^{-4}$ & $9.60560 \times 10^{-5}$ & $4.88417 \times 10^{-5}$ & $3.06953 \times 10^{-5}$ \\
1.7 & $4.56135 \times 10^{-4}$ & $2.12592 \times 10^{-4}$ & $1.25754 \times 10^{-4}$ & $4.36385 \times 10^{-5}$ & $2.02588 \times 10^{-5}$ & $1.19623 \times 10^{-5}$ \\
1.9 & $2.32443 \times 10^{-4}$ & $9.89421 \times 10^{-4}$ & $5.50011 \times 10^{-5}$ & $1.68449 \times 10^{-5}$ & $7.15737 \times 10^{-6}$ & $4.18048 \times 10^{-6}$ \\
\hline
\end{tabular}

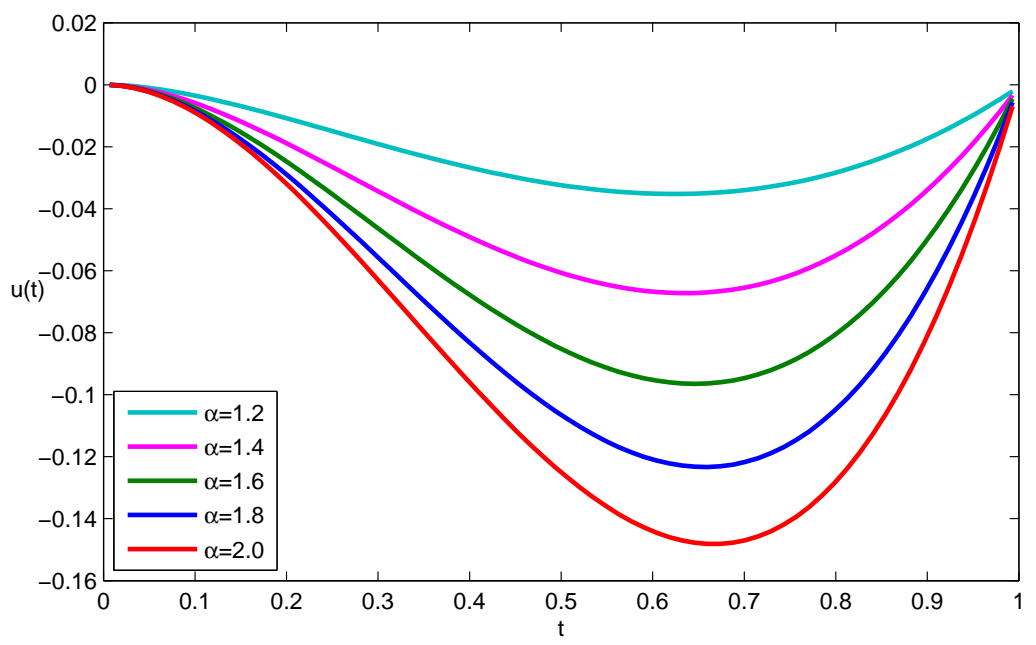

Figure 2. Numerical solutions for fixed values of $k=3, M=4$ and different values of $\alpha$.

Example 2. Consider fractional boundary value problem with variable coefficient

$$
\begin{gathered}
(1-t)^{2} \mathcal{D}_{t}^{\alpha} u(t)-(1-t) u(t)=1, \quad 1<\alpha \leq 2, \\
u(0)=1, \quad u(1)=\frac{1}{2} .
\end{gathered}
$$

The approximate solutions are obtained for different values of $\alpha$ by the Green-CAS method, as discussed in Section 3. For the case, when $\alpha=2$, the absolute error for $M=4$ and $k=9$ are presented in Table 2. Also, numerical results of proposed method are compared with the results of Haar wavelets method [30] and the method based on Taylor's expansion [48]. The comparison shows that the present method is quite satisfactory and competitive with other methods.

\begin{tabular}{|c|c|c|c|c|}
\hline & \multicolumn{2}{|c|}{ Method [48] } & Haar Wavelets [30] & Green-CAS \\
\hline 0.1 & $1.590477 \times 10^{-4}$ & $2.281673 \times 10^{-4}$ & $1.855711 \times 10^{-7}$ & $9.343142 \times 10^{-9}$ \\
\hline 0.2 & $1.047506 \times 10^{-4}$ & $2.469834 \times 10^{-4}$ & $1.030758 \times 10^{-7}$ & $7.580838 \times 10^{-9}$ \\
\hline 0.3 & $2.784706 \times 10^{-5}$ & $1.858469 \times 10^{-4}$ & $5.238972 \times 10^{-8}$ & $6.246745 \times 10^{-9}$ \\
\hline 0.4 & $4.550068 \times 10^{-5}$ & $1.157510 \times 10^{-4}$ & $2.279295 \times 10^{-8}$ & $5.196516 \times 10^{-9}$ \\
\hline 0.5 & $1.167467 \times 10^{-4}$ & $6.412753 \times 10^{-5}$ & $6.420743 \times 10^{-9}$ & $4.339887 \times 10^{-9}$ \\
\hline 0.6 & $1.812416 \times 10^{-4}$ & $3.403860 \times 10^{-5}$ & $2.670990 \times 10^{-9}$ & $1.618514 \times 10^{-9}$ \\
\hline 0.7 & $2.228086 \times 10^{-4}$ & $2.153643 \times 10^{-5}$ & $5.052727 \times 10^{-9}$ & $2.993625 \times 10^{-9}$ \\
\hline 0.8 & $2.213083 \times 10^{-4}$ & $1.719699 \times 10^{-5}$ & $1.285991 \times 10^{-8}$ & $2.438836 \times 10^{-9}$ \\
\hline 0.9 & $1.543950 \times 10^{-4}$ & $1.205234 \times 10^{-5}$ & $2.474367 \times 10^{-8}$ & $1.935839 \times 10^{-9}$ \\
\hline
\end{tabular}

Table 2. Absolute error for fixed values of $M=4$ and $k=9$. 
Example 3. Consider the Bagley-Torvik equation

$$
a(t) \mathcal{D}_{t}^{\alpha} u(t)+b(t) \mathcal{D}_{t}^{\beta} u(t)+c(t) u(t)=g(t), \quad 1<\alpha \leq 2,
$$

subject to the boundary conditions

$$
u(0)=u_{0}, \quad u(1)=u_{1} .
$$

For Bagley-Torvik equation we substitute the values $\alpha=2, \beta=1.5, a(t)=b(t)=c(t)=1$, and $g(t)=2+4 \sqrt{\frac{t}{\pi}}+t^{2}$ in (52). It can be conventionally verified that the exact solution to Equation (52) is $u(t)=t^{2}$. For numerical solutions at $\alpha=2$ and $\beta=1.5$, the algebraic matrix form of Equation (52) after applying Green-CAS method becomes

$$
C^{T}\left\{\Psi_{m \times m}+P_{m \times m}^{2-\beta} \Psi_{m \times m}+\hat{G}_{1 m \times m} \Psi_{m \times m}\right\}=F^{T},
$$

where $F^{T}$ is the evaluation of the function $f(t)=2+4 \sqrt{\frac{t}{\pi}}+t^{2}-t$ at the collocation points. The approximate solution of Equation (52) for the values $\alpha=2$ and $0<\beta \leq 1$, we get

$$
C^{T}\left\{\Psi_{m \times m}+\hat{G}_{3 m \times m} \Psi_{m \times m}+\hat{G}_{1 m \times m} \Psi_{m \times m}\right\}=H^{T} .
$$

where $H^{T}$ is a vector of the function $h(t)=2+\frac{3}{3-\beta} t^{2-\beta}+t^{2}-t$ at the collocation points. The absolute error

\begin{tabular}{|c|c|c|c|c|c|}
\hline \multirow{2}{*}{$t$} & \multicolumn{5}{|c|}{$\beta=1.5, \alpha=2$} \\
\hline & $M=3$ & $M=4$ & $M=5$ & $M=6$ & $M=7$ \\
\hline 0.2 & $7.74305 \times 10^{-5}$ & $4.58037 \times 10^{-5}$ & $3.00416 \times 10^{-5}$ & $2.11037 \times 10^{-5}$ & $1.55693 \times 10^{-5}$ \\
\hline 0.4 & $7.57783 \times 10^{-5}$ & $4.45792 \times 10^{-5}$ & $2.90871 \times 10^{-5}$ & $2.03323 \times 10^{-5}$ & $1.49287 \times 10^{-5}$ \\
\hline 0.6 & $7.61337 \times 10^{-5}$ & $4.49713 \times 10^{-5}$ & $2.94556 \times 10^{-5}$ & $2.06653 \times 10^{-5}$ & $1.52270 \times 10^{-5}$ \\
\hline \multirow[t]{2}{*}{0.8} & $7.76240 \times 10^{-5}$ & $4.63196 \times 10^{-5}$ & $306255 \times 10^{-5}$ & $2.16776 \times 10^{-5}$ & $1.61088 \times 10^{-5}$ \\
\hline & \multicolumn{5}{|c|}{$\beta=0.5, \alpha=2$} \\
\hline 0.2 & $2.06054 \times 10^{-4}$ & $1.33957 \times 10^{-4}$ & $9.52476 \times 10^{-5}$ & $7.18385 \times 10^{-5}$ & $5.64925 \times 10^{-5}$ \\
\hline 0.4 & $2.67027 \times 10^{-4}$ & $1.75371 \times 10^{-4}$ & $1.25682 \times 10^{-4}$ & $9.54010 \times 10^{-5}$ & $7.54226 \times 10^{-5}$ \\
\hline 0.6 & $2.61842 \times 10^{-4}$ & $1.71871 \times 10^{-4}$ & $1.23122 \times 10^{-4}$ & $9.34253 \times 10^{-5}$ & $7.38397 \times 10^{-5}$ \\
\hline 0.8 & $1.96070 \times 10^{-4}$ & $1.27224 \times 10^{-4}$ & $9.03248 \times 10^{-5}$ & $6.80420 \times 10^{-5}$ & $5.34518 \times 10^{-5}$ \\
\hline
\end{tabular}
is obtained from the exact and numerical solution at fixed values of $\alpha=2$ and different values of $\beta$ as shown in the tabular form in Table 3.

Table 3. Absolute error for $\alpha=2, k=3$ and different values of $\beta$ and $M$.

\subsection{Non-Linear Case}

The technique named quasilinearization was presented by Kalabas and Bellman [49] as a generalization of a specific method (Newton-Raphson) [43] which assist in solving the non-linear ordinary and partial differential equations. We briefly explain implementation procedure for the quasilinearization technique for linearizing non-linear fractional differential equations.

Let us consider the non-linear fractional boundary value problem

$$
\mathcal{D}_{t}^{\alpha} u(t)=g(t, u(t)), \quad 1<\alpha \leq 2, \quad t \in[0,1],
$$

subject to the boundary conditions $u(0)=\mu$ and $u(1)=\omega$.

Suppose the initial approximation of the function $u(t)$ is $u_{0}(t)$. Applying the quasilinearization technique about $u_{0}$ to Equation (56), we have

$$
\mathcal{D}_{t}^{\alpha}(t)=g\left(t, u_{0}(t)\right)+\left[u(t)-u_{0}(t)\right] g_{u_{0}}\left(t, u_{0}(t)\right) .
$$


which is a linear equation, solving (57) for $u(t)$, denoting it $u_{1}(t)$ and expanding (56) about $u_{1}(t)$, we have

$$
\mathcal{D}_{t}^{\alpha} u(t)=g\left(t, u_{1}(t)\right)+\left[u(t)-u_{1}(t)\right] g_{u_{1}}\left(t, u_{1}(t)\right),
$$

which is a third approximation. Assume that the iterative procedure is convergent. We continue the process till the desired accuracy is obtained. The recurrence relation can be written in the form

$$
\begin{aligned}
\mathcal{D}_{t}^{\alpha} u_{r+1}(t)= & g\left(t, u_{r}(t)\right)+\left[u_{r+1}(t)-u_{r}(t)\right] g_{u_{r}}\left(t, u_{r}(t)\right) . \\
& u_{r+1}(0)=\mu, \quad u_{r+1}(1)=\omega .
\end{aligned}
$$

which is a sequence of linear fractional differential equations and the function $u_{r}(t)$ is known function which can be used to find $u_{r+1}(t) \cong u(t)$.

Example 4. Temperature distribution equation.

Consider the following mathematical model which describes the temperature distribution in lumped system of combined convection-radiation in a slab made of materials with variable thermal conductivity

$$
\mathcal{D}_{t}^{\alpha} u(t)-\eta u^{4}(t)=0, \quad 1<\alpha \leq 2, \quad t \in[0,1],
$$

subject to the boundary conditions $u^{\prime}(0)=0$ and $u(1)=1$, where $u=\frac{T-T_{a}}{T_{i}-T_{a}}$ and $t=\frac{x}{V \rho c_{a} / A h}$ are dimensionless temperature and time respectively and $\eta=\left(T-T_{a}\right) \beta$, where $V, A, \rho, c, T_{i}, T_{a}, c_{a}$ and $h$ are the volume, surface area, density, specific heat, the initial temperature, temperature of the convection environment, specific heat at temperature $T_{a}$ and heat transfer coefficient of the lumped system, respectively.

Using quasilinearization technique we get the linear fractional differential equation of the form

$$
\mathcal{D}_{t}^{\alpha} u_{n+1}(t)=4 \eta u_{n}^{3}(t) u_{n+1}(t)-3 \eta u_{n}^{4}(t),
$$

with boundary conditions $u_{n+1}^{\prime}(0)=0$ and $u_{n+1}(1)=1$. This boundary value problem is solved by the procedure discuss in Section 3. Table 4 contains the numerical solutions achieved by the Green-CAS along the method discuss in $[39,40]$. It indicates that the results obtained by proposed method are quite better. We compare our method with numerical solution provided by MAPLE [40] and generalized approximation method $y_{G A}$ [39] and homotopy perturbation method $y_{H P M}$ [39]. The numerical solutions for different values of $\eta$ and $\alpha$ are documented in the graphical form in the Figure 3. Also Figure 4 shows the numerical solutions for $k=3$, $M=4, \eta=1.5$ and different values of $\alpha$.

Table 4. Numerical solutions for fixed values of $\alpha=2, M=10$ and $k=7$.

\begin{tabular}{ccccc}
\hline $\boldsymbol{\eta}=\mathbf{0 . 6}$ & & & & \\
\hline $\boldsymbol{t}$ & Maple [40] & $\boldsymbol{Y}_{\mathrm{GA}}[39]$ & $\boldsymbol{Y}_{\text {HPM }}[39]$ & $\boldsymbol{G}_{\mathrm{CAS}}$ \\
\hline 0.0 & 0.834542 & 0.963536 & 0.640000 & 0.834430 \\
0.2 & 0.840390 & 0.964009 & 0.652096 & 0.840307 \\
0.4 & 0.858269 & 0.965742 & 0.689536 & 0.858211 \\
0.6 & 0.889247 & 0.969893 & 0.755776 & 0.889211 \\
0.8 & 0.935346 & 0.979233 & 0.866576 & 0.935328 \\
\hline $\boldsymbol{\eta}=\mathbf{2 . 0}$ & & & & \\
\hline 0.0 & 0.694318 & 0.968771 & -0.666667 & 0.694212 \\
0.2 & 0.703698 & 0.968804 & -0.625600 & 0.703636 \\
0.4 & 0.732894 & 0.969008 & -0.489600 & 0.732860 \\
0.6 & 0.785488 & 0.970024 & -0.220267 & 0.785471 \\
0.8 & 0.869161 & 0.975059 & -0.246400 & 0.869156 \\
\hline
\end{tabular}



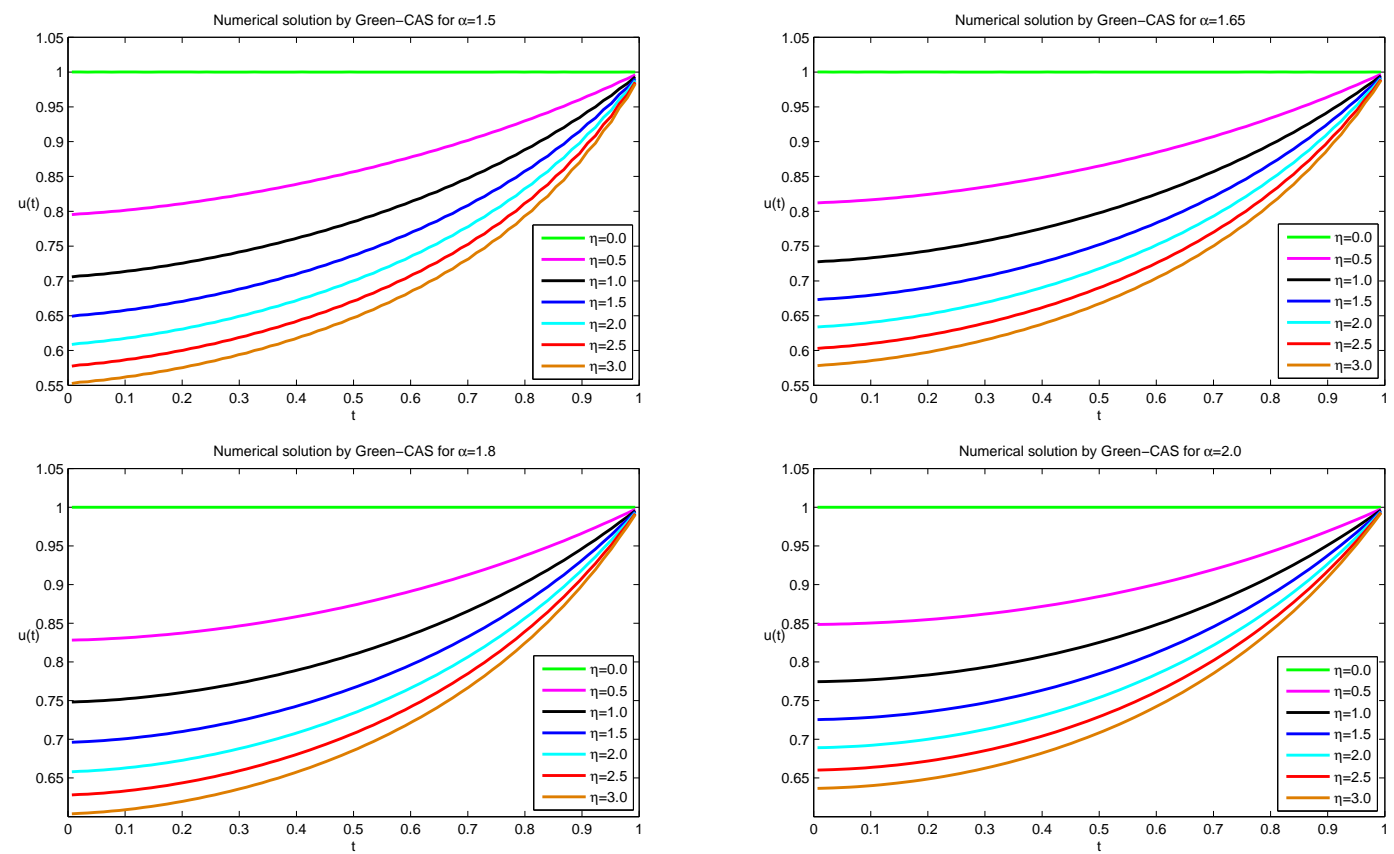

Figure 3. Numerical solutions of Equation (61) by Green-CAS for $k=3, M=4$ and different fixed values of $\alpha$ and $\eta$.

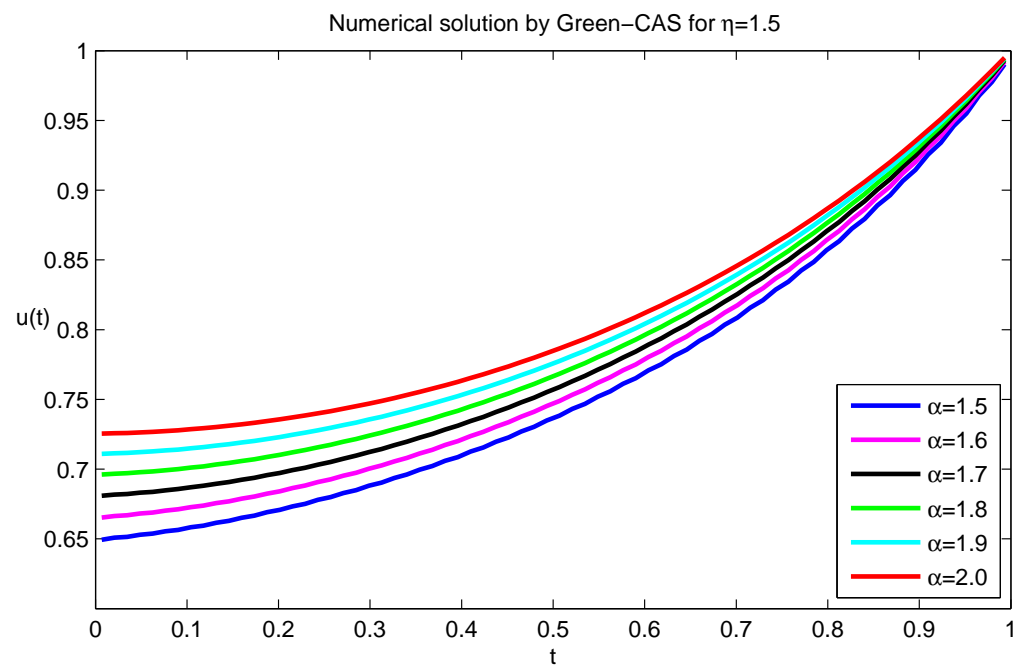

Figure 4. Numerical solutions of Equation (61) by Green-CAS for $k=3, M=4 \eta=1.5$ and different values of $\alpha$.

Example 5. Consider the non-linear oscillator equation

$$
\mathcal{D}_{t}^{\alpha} u(t)-u(t)+[u(t)]^{2}+\left[\mathcal{D}_{t}^{\beta} u(t)\right]^{2}-1=0, \quad 1<\alpha \leq 2,0<\beta \leq 1,
$$

subject to the boundary conditions

$$
u(0)=2, \quad u(1)=1+\cos (1)
$$

For $\alpha=2$ and $\beta=1$ the exact solution of Equation (63) is $u(t)=1+\cos (t)$. Now applying the quasilinearization to the (63), we get the sequence of linear fractional differential equation as

$$
\mathcal{D}_{t}^{\alpha} u_{n+1}(t)-\left(1-2 u_{n}(t)\right) u_{n+1}(t)+2 \mathcal{D}_{t}^{\beta} u_{n}(t) \mathcal{D}_{t}^{\beta} u_{n+1}(t)=1+\left[u_{n}(t)\right]^{2}+\left[\mathcal{D}_{t}^{\beta} u_{n}(t)\right]^{2}, \quad 1<\alpha \leq 2,0<\beta \leq 1,
$$


with $u_{n+1}(0)=2, u_{n+1}(1)=1+\cos (1)$. The Equation (65) is linear and for numerical solution we use the method which is discussed in Section 3. In Table 5 the absolute error of the exact and numerical solutions for different values of $k$ and $M$ are presented. We observe that the error decrease by increasing the values of $k$ and $M$, as promised in convergence analysis. The numerical solutions are shown in the Figure 5 for different values of $\alpha$ which tend to the exact solution when $\alpha$ approaches 2 .

Table 5. Absolute error for fixed values of $\alpha=2, \beta=1$ and different values of $k$ and $M$.

\begin{tabular}{ccccc}
\hline & \multicolumn{2}{c}{$k=3$} & \multicolumn{2}{c}{$k=5$} \\
\cline { 2 - 5 } $\boldsymbol{n}$ & $\boldsymbol{M}=\mathbf{3}$ & $\boldsymbol{M}=\mathbf{5}$ & $\boldsymbol{M}=\mathbf{3}$ & $\boldsymbol{M}=\mathbf{5}$ \\
\hline 0.1 & $2.85045 \times 10^{-4}$ & $1.93246 \times 10^{-4}$ & $8.08870 \times 10^{-5}$ & $5.22195 \times 10^{-5}$ \\
0.2 & $5.90695 \times 10^{-4}$ & $3.89928 \times 10^{-4}$ & $1.59085 \times 10^{-4}$ & $102123 \times 10^{-4}$ \\
0.3 & $8.56315 \times 10^{-4}$ & $5.60635 \times 10^{-4}$ & $2.26869 \times 10^{-4}$ & $1.45369 \times 10^{-4}$ \\
0.4 & $1.06161 \times 10^{-3}$ & $6.92388 \times 10^{-4}$ & $2.79110 \times 10^{-4}$ & $1.78686 \times 10^{-4}$ \\
0.5 & $1.18694 \times 10^{-3}$ & $7.72592 \times 10^{-4}$ & $3.10820 \times 10^{-4}$ & $1.98898 \times 10^{-4}$ \\
0.6 & $1.21341 \times 10^{-3}$ & $7.89112 \times 10^{-4}$ & $3.17186 \times 10^{-4}$ & $2.02931 \times 10^{-4}$ \\
0.7 & $1.12292 \times 10^{-3}$ & $7.30297 \times 10^{-4}$ & $2.93571 \times 10^{-4}$ & $1.87827 \times 10^{-4}$ \\
0.8 & $8.98148 \times 10^{-4}$ & $5.84954 \times 10^{-4}$ & $2.35505 \times 10^{-4}$ & $1.50731 \times 10^{-4}$ \\
0.9 & $5.22364 \times 10^{-4}$ & $3.42238 \times 10^{-4}$ & $1.38639 \times 10^{-4}$ & $8.88610 \times 10^{-5}$ \\
\hline
\end{tabular}

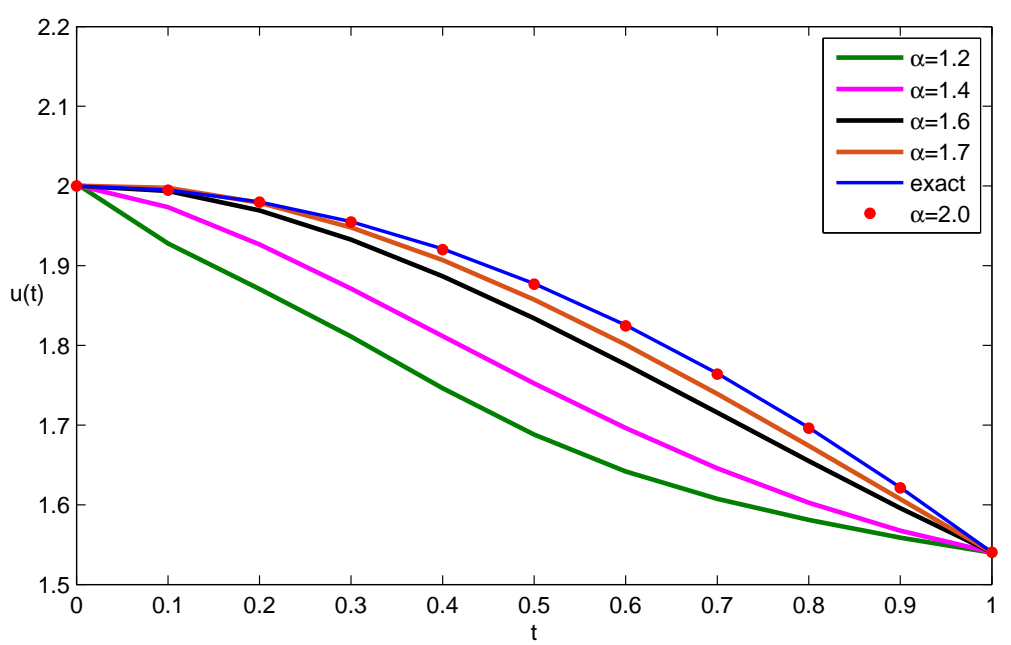

Figure 5. Numerical and exact solutions for fixed values of $K=3, M=4$ and different values of $\alpha$.

\section{Error Analysis}

In this part, we derive inequality in context of upper bound which shows the convergence of Green-CAS method. The convergence of CAS wavelet is given in [40]. By following the similar procedure, we extend the analysis for the present Green-CAS method.

Theorem 2. Suppose that the $u_{r+1}^{\prime \prime}(t)$ is continuous and bounded on $[0,1]$ that is there exist $\gamma>0\left|u_{r+1}^{\prime \prime}(t)\right| \leq$ $\gamma \forall t \in[0,1]$. and also assume that $u_{r+1}^{k, M}(t)$ is approximation of $u_{r+1}(t)$, then we have

$$
\left|u_{r+1}^{k, M}(t)-u_{r+1}(t)\right| \leq \frac{\gamma N}{\pi^{2} \Gamma(\alpha+1)} \sum_{n=2^{k}}^{\infty} \sum_{\hat{m}=M+1}^{\infty} \frac{1}{(n+1)^{\frac{5}{2}} \hat{m}^{2}} .
$$

Proof. Consider the non-linear fractional boundary value problems

$$
\mathcal{D}_{t}^{\alpha} u(t)=f(u(t), t), \quad t \in[0,1],
$$


with boundary conditions $u(0)=u_{0}, u(1)=u_{1}$. The integral representation of above Equation (66) after using boundary conditions, can be written as

$$
u(t)=\int_{0}^{1} G(t, \xi) f(u(\xi), \xi) d \xi+h(t)
$$

where $h(t)=\left(u_{1}-u_{0}\right) t+u_{0}$. Applying the quasilinearization technique to Equation (67), we have

$$
u_{r+1}(t)=g(t)+\int_{0}^{1} f_{u_{r}}\left(u_{r}(\xi), \xi\right) G(t, \xi) u_{r+1}(\xi) d \xi,
$$

where $g(t)=h(t)+\int_{0}^{1} G(t, \xi)\left[f\left(u_{r}(\xi), \xi\right)-u_{r}(\xi) f_{u_{r}}\left(u_{r}(\xi), \xi\right)\right] d \xi$ and $u_{r}(t)$ is known and can be used to obtained $u_{r+1}(t)$. Let $u_{r+1}^{k, M}(t)$ be the approximation of $u_{r+1}(t)$, by Green-CAS method, then we have

$$
u_{r+1}^{k, M}(t) \approx g(t)+\int_{0}^{1} f_{u_{r}}\left(u_{r}(\xi), \xi\right) G(t, \xi) u_{r+1}^{k, M}(\xi) d \xi,
$$

From Equations (68) and (69), we have

$$
\left|u_{r+1}^{k, M}(t)-u_{r+1}(t)\right| \leq \int_{0}^{1}\left|f_{u_{r}}\left(u_{r}(\xi), \xi\right)\right| G(t, \xi)\left|u_{r+1}^{k, M}(t)-u_{r+1}(t)\right| d \xi .
$$

Therefore, from the Lemma 3 we have,

$$
\left|u_{r+1}^{k, M}(t)-u_{r+1}(t)\right| \leq \frac{\gamma N}{\pi^{2}} \sum_{n=2^{k}}^{\infty} \sum_{\hat{m}=M+1}^{\infty} \frac{1}{(n+1)^{\frac{5}{2}} \hat{m}^{2}}\left|\int_{0}^{1} G(t, \xi) d \xi\right| .
$$

Letting $N=\max \left|f_{u_{r}}\left(u_{r}(\xi), \xi\right)\right|$. The value of $\int_{0}^{1} G(t, \xi) d \xi$ is

$$
\begin{gathered}
\int_{0}^{1} G(t, \xi) d \xi=\frac{1}{\Gamma(\alpha)}\left\{\int_{0}^{t}\left[(t-\xi)^{\alpha-1}-t(1-\xi)^{\alpha-1}\right] d \xi-t \int_{t}^{1}(1-\xi)^{\alpha-1} d \xi\right\} \\
=\frac{1}{\Gamma(\alpha+1)}\left(t^{\alpha}-t\right),
\end{gathered}
$$

as $-t \leq 0$ since $t \in[0,1]$. Therefore

$$
\int_{0}^{1} G(t, \xi) d \xi \leq \frac{t^{\alpha}}{\Gamma(\alpha+1)} \leq \frac{1}{\Gamma(\alpha+1)} .
$$

Therefore, using (72) into (71), we obtain

$$
\left|u_{r+1}^{k, M}(t)-u_{r+1}(t)\right| \leq \frac{\gamma N}{\pi^{2} \Gamma(\alpha+1)} \sum_{n=2^{k}}^{\infty} \sum_{\hat{m}=M+1}^{\infty} \frac{1}{(n+1)^{\frac{5}{2}} \hat{m}^{2}} .
$$

\section{Conclusions}

A novel method named Green-CAS method has been developed for solving linear and non-linear fractional differential equations with boundary conditions. One of the advantages of this method is that it is considerably simple and easy to implement. To check the reliability and accuracy of the method we tested the method on different physical and hypothetical models. According to Table 4, our results are better than generalized approximation method and homotopy perturbation method, and are in good agreement with exact solution. 
The method may be extended to solve boundary value problems for partial differential equations and fractional differential equations appearing in mathematical modeling and engineering applications.

Author Contributions: Author's contributions are as under: U.S.: Conceptualization, Investigation, Visualization; M.I.: Software, Investigation, original draft preparation, Data; J.A.: Funding acquisition, Validation, curation, review and editing, Project administration; M.u.R.: Supervision, Methodology, Formal analysis.

Funding: Prince Sultan University for funding this work through research group Non-Linear Analysis Methods in Applied Mathematics (NAMAM) group number RG-DES-2017-01-17.

Acknowledgments: The third author would like to thank Prince Sultan University for funding this work through research group Non-Linear Analysis Methods in Applied Mathematics (NAMAM) group number RG-DES-2017-01-17.

Conflicts of Interest: The authors declare no conflict of interest.

\section{References}

1. He, J.H. A new approach to nonlinear partial differential equations. Commun. Nonlinear Sci. Numer. Simul. 1997, 2, 230-235. [CrossRef]

2. He, J.H. Approximate analytical solution for seepage flow with fractional derivatives in porous media. Comput. Methods Appl. Mech. Eng. 1998, 167, 57-68. [CrossRef]

3. He, J.H. Some asymptotic methods for strongly nonlinear equations. Int. J. Mod. Phys. B 2006, 20, 1141-1199. [CrossRef]

4. Anh, V.V.; Leonenko, N.N. Spectral analysis of fractional kinetic equations with random data. J. Stat. Phys. 2001, 104, 1349-1387. [CrossRef]

5. Blaszczyk, T.; Ciesielski, M.; Klimek, M.; Leszczynski, J. Numerical solution of fractional oscillator equation. Appl. Math. Comput. 2011, 218, 2480-2488. [CrossRef]

6. Khan, N.A.; Ara, A.; Ali, S.A.; Mahmood, A. Analytical study of Navier-Stokes equation with fractional orders using He's homotopy perturbation and variational iteration methods. Int. J. Nonlinear Sci. Numer. Simul. 2009, 10, 1127-1134. [CrossRef]

7. Schulz, B.M.; Schulz, M. Numerical investigations of anomalous diffusion effects in glasses. J. Non-Cryst. Solids 2006, 352, 4884-4887. [CrossRef]

8. Burgos, C.; Cortés, J.C.; Villafuerte, L.; Villanueva, R.J. Mean square calculus and random linear fractional differential equations: Theory and applications. Appl. Math. Nonlinear Sci. Appl. Math. Nonl. Sci. 2017, 2, 317-328. [CrossRef]

9. Youssef, I.K.; el Dewaik, M.H. Solving Poisson's Equations with fractional order using Haarwavelet. Appl. Math. Nonlinear Sci. Appl. Math. Nonlinear Sci. 2017, 2, 271-284. [CrossRef]

10. Guirao, J.L.G.; Bayram, M. Computational Methods in Applied Analysis and Mathematical Modeling; MDPI: Basel, Switzerland, 2019; ISSN 2227-7390.

11. He, J.H. Some applications of nonlinear fractional differential equations and their approximations. Bull. Sci. Technol. Soc. 1999, 15, 86-90.

12. Magin, R.L. Fractional calculus in bioengineering. Redd. Begell House 2006, 32, 105-194.

13. Engheta, N. On fractional calculus and fractional multipoles in electromagnetism. IEEE Trans. Antennas Propag. 1996, 44, 554-566. [CrossRef]

14. Sierociuk, D.; Dzielinski, A.; Sarwas, G.; Petras, I.; Podlubny, I.; Skovranek, T. Modelling heat transfer in heterogeneous media using fractional calculus. Philos. Trans. R. Soc. A Math. Phys. Eng. Sci. 2013, 371, 20120146. [CrossRef] [PubMed]

15. Cosenza, P.; Korosak, D. Secondary consolidation of clay as an anomalous diffusion process. Int. J. Numer. Anal. Methods Geomech. 2014, 38, 1231-1246. [CrossRef]

16. Pagnini, G. Erdelyi-Kober fractional diffusion. Fract. Calc. Appl. Anal. 2012, 15, 117-127. [CrossRef]

17. Martinez-Garcia, M.; Gordon, T. Human control of systems with fractional order dynamics. In Proceedings of the IEEE International Conference on Systems, Man, and Cybernetics (SMC), Budapest, Hungary, 9-12 October 2016.

18. Martinez-Garcia, M.; Gordon, T.; Shu, L. Extended crossover model for human-control of fractional order plants. IEEE Access 2017, 5, 27622-27635. [CrossRef] 
19. Li, B.C.; Luo, J.S. Wavelet Analysis and Its Application; Electronic Industrial Publication: Beijing, China, 2005.

20. Yousefi, S.; Banifatemi, A. Numerical solution of Fredholm integral equations by using CAS wavelets. Appl. Math. Comput. 2006, 183, 458-463. [CrossRef]

21. Yi, M.; Huang, J. CAS wavelet method for solving the fractional integro-differential equation with a weakly singular kernel. Int. J. Comput. Math. 2014, 92, 1715-1728. [CrossRef]

22. Shamooshaky, M.M.; Assari, P.; Adibi, H. CAS wavelet method for the numerical solution of boundary integral equations with logarithmic singular kernels. Int. J. Math. Model. Comput. 2014, 4, 377-987.

23. Saeedi, H.; Moghadam, M.M. Numerical solution of nonlinear Volterra integro-differential equations of arbitrary order by CAS wavelets. Commun. Nonlinear Sci. Numer. Simul. 2011, 16, 1216-1226. [CrossRef]

24. Saeed, U. CAS Picard method for fractional nonlinear differential equation. Appl. Math. Comput. 2017, 307, 102-112. [CrossRef]

25. Aziz, I.; Khan, W. Numerical integration of multi-dimensional highly oscillatory, gentle oscillatory and non-oscillatory integrands based on wavelets and radial basis functions. Eng. Anal. Bound. Elem. 2012, 36, 1284-1295.

26. Aziz, I.; Fayyaz, M. A new approach for numerical solution of integro-differential equations via Haar wavelets. Int. J. Comput. Math. 2013, 90, 1971-1989.

27. Saeed, U.; Rehman, M. Haar wavelet-quasilinearization technique for fractional nonlinear differential equations. Appl. Math. Comput. 2013, 220, 630-648. [CrossRef]

28. Wu, J.L. A wavelet operational method for solving fractional partial differential equations numerically. Appl. Math. Comput. 2009, 214, 31-40. [CrossRef]

29. Lepik, Ü. Numerical solution of evolution equations by the Haar wavelet method. Appl. Math. Comput. 2007, 185, 695-704. [CrossRef]

30. Rehman, M.; Khan, R.A. A numerical method for solving boundary value problems for fractional differential equations. Appl. Math. Model. 2012, 36, 894-907. [CrossRef]

31. Diaz, L.A.; Martin, M.T.; Vampa, V. Daubechies wavelet beam and plate finite elements. Finite Elem. Anal. Des. 2009, 45, 200-209. [CrossRef]

32. Dehghan, M.; Lakestani, M. Numerical solution of nonlinear system of second-order boundary value problems using cubic B-spline scaling functions. Int. J. Comput. Math. 2008, 85, 1455-1461. [CrossRef]

33. Rehman, M.; Khan, R.A. The Legendre wavelet method for solving fractional differential equations. Commun. Nonlinear Sci. Numer. Simul. 2011, 16, 4163-4173. [CrossRef]

34. Wang, X.T. Numerical solution of time-varying systems with a stretch by general Legendre wavelets. Appl. Math. Comput. 2008, 198, 613-620. [CrossRef]

35. Khan, H.; Arif, M.; Mohyud-Din, S.T. Numerical Solution of Fractional Boundary Value Problems by Using Chebyshev Wavelet. Matrix Sci. Math. 2019, 3, 13-26. [CrossRef]

36. Akgül, A.; Akgül, E.K. A Novel Method for Solutions of Fourth-Order Fractional Boundary Value Problems. Fractal Fract. 2019, 3, 33. [CrossRef]

37. Gholami, S.; Babolian, E.; Javidi, M. Fractional pseudospectral integration/differentiation matrix and fractional differential equations. Appl. Math. Comput. 2019, 343, 314-327. [CrossRef]

38. Patricio, M.F.S.; Ramos, H.; Patricio, M. Solving initial and boundary value problems of fractional ordinary differential equations by using collocation and fractional powers. J. Comput. Appl. Math. 2019, 354, 348-359. [CrossRef]

39. Khan, R.A. Generalized approximation method for heat radiation equations. Appl. Math. Comput. 2009, 212, 287-295. [CrossRef]

40. Saeed, U. Assessment of Haar wavelet-quasilinearization technique in heat convection-radiation equations. Appl. Comput. Intell. Soft Comput. 2014. [CrossRef]

41. Diethelm, K. The Analysis of Fractional Differential Equations; Lecture Notes in Mathematics 2004; Springer: Berlin/Heidelberg, Germany, 2010. [CrossRef]

42. Kilicman, A.; al Zhour, Z.A.A. Kronecker operational matrices for fractional calculus and some applications. Appl. Math. Comput. 2007, 187, 250-265. [CrossRef]

43. Conte, S.D.; de Boor, C. Elementary Numerical Analysis; SIAM: Philadelphia, PA, USA, 2018.

44. Diethelm, K. An extension of the well-posedness concept for fractional differential equations of Caputo's type. Appl. Anal. 2014, 93, 2126-2135. [CrossRef] 
45. Su, X.; Zhang, S. Solutions to boundary-value problems for nonlinear differential equations of fractional order. Electr. J. Differ. Equ. 2009, 2009, 1-15.

46. $\mathrm{Su}, \mathrm{X}$. Boundary value problem for a coupled system of nonlinear fractional differential equations. Appl. Math. Lett. 2009, 22, 64-69. [CrossRef]

47. Diethelm, K.; Ford, N.J. Analysis of fractional differential equations. J. Math. Anal. Appl. 2002, 265, 229-248. [CrossRef]

48. Li, X.F. Approximate solution of linear ordinary differential equations with variable coefficients. Math. Comput. Simul. 2007, 75, 113-125. [CrossRef]

49. Bellman, R.E.; Kalaba, R.E. Quasilinearization and Nonlinear Boundary-Value Problems; Elsevier Publishing Company: New York, NY, USA, 1965.

(C) 2019 by the authors. Licensee MDPI, Basel, Switzerland. This article is an open access article distributed under the terms and conditions of the Creative Commons Attribution (CC BY) license (http://creativecommons.org/licenses/by/4.0/). 